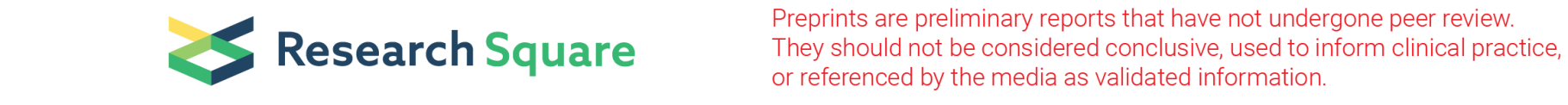

\title{
Effectiveness of mobile applications in diabetic patients' healthy lifestyles: review of systematic reviews.
}

Francisco Jesús Represas Carrera ( $\square$ franciscorepresascarrera@gmail.com )

Primary health care. Vigo Sanitary Area. Galician Health Service (SERGAS). Galicia South Research Institute, Vigo, Spain. https://orcid.org/0000-0003-0419-4570

Ángel Alfredo Martínez Ques

Primary health care. Ourense Sanitary Area. Galician Health Service (SERGAS). Galicia Southern Research Institute, Ourense, Spain. https://orcid.org/0000-0002-1034-0397

\section{Ana Clavería Fontán}

Primary health care. Vigo Sanitary Area. Galician Health Service (SERGAS). Galicia South Research Institute, Vigo. Primary Care Research Network, Spain. https://orcid.org/0000-0001-9552-1260

\section{Systematic Review}

Keywords: Diabetes Mellitus, Systematic Review, Life Style, Mobile Applications

Posted Date: November 24th, 2020

DOI: https://doi.org/10.21203/rs.3.rs-115192/v1

License: (c) (i) This work is licensed under a Creative Commons Attribution 4.0 International License. Read Full License 


\section{Abstract}

Background: Diabetes mellitus (DM) is currently a major public health problem worldwide. It is traditionally approached by a clinical inpatient relationship between patients and healthcare professionals. However, the rise in of the use of new technologies, particularly mobile applications, is revolutionizing the traditional healthcare model with the introduction of telehealthcare.

Objective: (1) Examine the mobile applications that address lifestyles to improve the metabolic control of adult patients with Diabetes Mellitus. (2) Describe the characteristics of the used mobile applications, identify the healthy lifestyles they target, and describe any of their adverse effects.

Methods: Review of systematic reviews following Cochrane Collaboration and Joanna Briggs Institute guidelines. We included studies that used any mobile application to help patients improve DM self-management by focusing on healthy lifestyles. Studies needed to include a control group receiving regular care without using mobile devices. In May 2018, Medline, Embase, Cochrane, LILACS, PsychINFO, Cinahl and Science Direct were searched, updated in May 2019. The methodological quality of the studies was assessed by the Amstar-2 tool.

Results: Seven systematic reviews of 798 articles were initially selected for the analysis. Interventions lasted 1-12 months. Twenty-three different mobile applications were identified. They were all related to eating and physical activity. Significant changes were found in HbA1c values, body weight and BMI, but no clear improvement was observed in others like lipid profile, quality of life or blood pressure. No significant adverse effects were identified.

Conclusions: Clearly evidence appeared for using mobile applications to improve glycemic control in diabetic patients in the short term, but not for long-term benefits. Thus carrying out further studies is necessary to learn about the long-term effectiveness of mobile applications to promote DM patients' healthy lifestyles.

\section{PROSPERO Register: CRD42019133685}

\section{Introduction}

The International Diabetes Federation (IDF) estimates some 425 million people diagnosed with diabetes mellitus (DM) worldwide, whose prevalence will rise to 693 million by 2045 [1]. It is well-known that DM significantly increases cardiovascular risk and is usually associated with unhealthy lifestyle; e.g. being overweight, obesity, smoking, unbalanced diet, sedentary lifestyle [2,3]. For these reasons, DM is currently a major public health problem worldwide [4].

The rapid progress in information technology in recent years has enabled the creation of software extensions, popularly known as mobile applications (Apps), that users can add to their mobile devices (Apps). These tools are being increasingly employed to manage different health matters. In 2018, more than 100,000 such applications were available to make users' health self-care easier [5].

The main aim of treating DM is to prevent or delay the onset of microvascular and macrovascular complications due to suitable metabolic control, where practicing healthy lifestyles is fundamental [6]. This therapeutic objective has been dealt with in the conventional health context by the doctor-patient relationship. At present, the mobile apps used for the same purpose are revolutionizing this traditional care model.

Given the substantial progress made in recent years in non face-to-face healthcare, in 2011 the World Health Organization (WHO) defined the " $m$-health" concept as the practice of medicine and public health supported by the use of mobile devices (mobile phones, patient monitoring devices, personal digital assistants and other wireless devices) [7]. Mobile systems allow the following: prevention, diagnosis and/or treatment activities also serve as a means of communication between healthcare team members, professionals and patients, and patient to patient [8].

The systematic reviews published so far about the effectiveness of Apps for promoting healthy lifestyles have yielded mixed and inconclusive results. Some reviews offer positive results [9-13], and the majority concludes that more research studies are necessary to assess their real effectiveness [14-25], while a few reviews state that these types of intervention are not effective [26,27].

Moreover, differences in facilitating elements and barriers have been described with the population using digital health platforms, which means that their accessibility and acceptability are not universal [28]. Accordingly, as part of its Action Plan on electronic health (eHealth) $2012-2020$ and the Digital Agenda, the European Commission has published the "Green Paper on mobile health", with which it intends to conduct a public consultation on current obstacles and problems related to implementing mobile health to help it reach its full potential [29].

The present review uses the "DM self-control" concept defined by Powers et al. [30] as the ongoing "process of facilitating the knowledge, skills and capacity necessary for diabetes self-care". Its ultimate goal is to improve clinical outcomes, health status and patients' quality of life. It 
also attempts to answer the question: are mobile applications that deal with lifestyles to improve the metabolic control of adult patients with DM effective?

In line with this question, we consider that the main objective is to examine mobile applications that address lifestyles to improve the metabolic control of adult patients with DM. As secondary objectives, we aim to describe the characteristics of the used apps, identify the healthy lifestyle aspect they target, and describe any adverse effects of their uses.

\section{Methods}

\section{Design}

A review of systematic reviews was performed. This design allowed us to compare and verify the findings of relevant reviews in response to similar review questions, which facilitates a view and clear understanding of a broad theme area [31]. Its main purpose is to summarize evidence from many research sources. Compared to a systematic review limited to one treatment or one intervention, this type of review offers a broader view of many interventions. It is useful for health-related technology assessments whose objective is to inform about patterns for clinical practice where many handling options must be considered and evaluated.

To implement the review, we followed the Cochrane Collaboration [32] and Reviewers Manual of the Joanna Briggs Institute [33] guidelines. To prepare this report, we took into account the PRISMA proposal [34] recommendations.

The protocol of this systematic review was registered in PROSPERO with code CRD42019133685 [35].

\section{Inclusion and exclusion criteria}

1. Type of study: Systematic review and/or meta-analysis.

2. Population: Patients aged over 18 years diagnosed with DM regardless of the type of treatment followed.

3. Intervention: using any App to help patients improve DM self-management by a healthy lifestyles approach. The mobile application can be used exclusively or combined with other types of interventions. The study needed to describe the type of employed mobile application and the addressed lifestyle aspect. Studies in which text messages were sent via mobile phones, independently of the use of any mobile application being excluded.

4. Comparison: a control group receiving regular care without using mobile devices toward healthier lifestyles.

5. Outcome measures. Primary: Glycosylated hemoglobin (HbA1c), change in body weight or body mass index (BMI), and occurrence of/increase in adverse effects (anxiety, depression, hypoglycemia episodes, etc.). Secondary: health-related quality of life (HRQOL), systolic (SBP) and diastolic (DBP) blood pressure, fasting blood glucose, lipid profile; total cholesterol (TC), LDL cholesterol (LDL-C), HDL cholesterol (HDL-C), triglycerides (TG).

The studies that did not report any primary or secondary outcomes were excluded. Studies had to include a comparative analysis of the outcomes measured at the baseline and those measured at the end of the intervention.

\section{Sources of information and search strategy}

To define the search strategy, we used a set of studies that was first divided to facilitate the location and definition of the main descriptors. The key terms and their synonyms were identified in the Medline (via PubMed), Cinahl and Google Scholar databases. The identified terms were combined to calibrate a search syntax in Medline by bearing the sensitivity and specificity criteria in mind.

Next a second search using all the identified keywords and index terms was performed in the following electronic databases: Medline, Embase, Cochrane, Lilacs, PsychINFO, Cinahl and Science Direct. Articles were selected in May 2018 and updated in May 2019. There were no limits in terms of language or year of publication. The syntax of the bibliographic search carried out in Medline is attached (Multimedia Appendix 1).

The search for unpublished studies was carried out in: Open Grey, ProQuest Dissertations \& Theses Global, Theseo, Networked Digital Library of Theses and Dissertations (NDLTD).

Finally, the reference lists of all the identified reports and articles were searched for additional studies. The search was completed by hand searching and reverse searching in reference journals specializing in smoking, diabetes and e-health.

The Mendeley reference management software was used to sort the bibliographic citations obtained in the search to eliminate duplicate citations and to order all the studies to facilitate the analysis.

\section{Study Selection}


The complete study selection process was carried out by peer review. In the event of disagreement between the two reviewers, a third party was invited to participate.

First, a selection of the studies was made based on their title and/or Abstract according to the predefined inclusion criteria. Duplicates and those articles which, given their title and/or Abstract did not match our field of interest, were eliminated. None of the authors of the review was blinded to the titles of journals or the authors or institutions of the study.

Second, a new selection was made by reading the full articles and verifying that they met the inclusion criteria.

\section{Assessing methodological quality}

After selection, studies were assessed as to their methodological quality with the Measurement Tool to Assess Systematic Reviews-2 (Amstar-2) [36].

Amstar-2 allows the assessment of systematic reviews, which include randomized controlled clinical trials (RCTs) and non randomized health interventions. This questionnaire consists of 16 items with several response categories: "yes", when the answer is positive; "no", when the item is not present or contains insufficient information to respond; "partial yes", when the item is partially present. Seven domains (items 2, 4, 7, 9, 11, 13 and 15) are considered critical because they can significantly affect a review's validity and conclusions. It classifies the quality of systematic reviews into four levels: high, moderate, low, very low.

This methodological quality assessment was made in pairs. Although provided for in the protocol, it was not necessary to consult a third reviewer because an agreement between reviewers was reached. We included reviews of moderate or high methodological quality in our review. Reviews with low or very low quality were excluded so as not to distort the evidence of the conclusions.

\section{Data extraction and variables}

The data from the studies were extracted, synthesized and recorded on an Excel sheet which included: Amstar-2 items, first author, year, source, search strategy, number of included studies, total number of participants, study design, intervention time (months) and outcome measures.

Data were extracted independently by two reviewers. The reliability and quality of the extracted data were ensured by cross-checking, rereading the complete studies and reviewing the collected data.

\section{Data analysis and synthesis}

After using the form to collect and synthesize all the relevant data, they were presented, depending on how they were reported in each case, in the form of mean, standard deviation, and/or $95 \%$ confidence interval with their statistical significance value $(P)$.

Descriptive results were presented according to the following sections: characteristics and quality of studies, Apps and lifestyle they intended to modify, primary outcome measures, secondary outcome measures and adverse effects of interventions.

\section{Results}

The initial bibliographic search yielded 798 articles, of which 23 duplicates were eliminated. Subsequently, 56 articles were selected for title and Abstract. After their complete reading, 39 were discarded because they did not meet the inclusion criteria. Finally, 17 articles were selected, of which 10 were discarded for their low methodological quality (Table 1). Thus the final selection included seven systematic reviews (Figure 1). 
Table 1. Quality assessment of systematic reviews through Amstar-2

\begin{tabular}{|c|c|c|c|c|c|c|c|c|c|c|c|c|c|c|c|c|c|}
\hline $\begin{array}{c}\text { ítems } \\
\text { Amstar-2 }\end{array}$ & 1 & 2 & 3 & 4 & 5 & 6 & 7 & 8 & 9 & 10 & 11 & 12 & 13 & 14 & 15 & 16 & $\begin{array}{l}\text { Methodological } \\
\text { Quality }\end{array}$ \\
\hline $\begin{array}{ll}\text { McMillan, } & 2017 \\
{[13]}\end{array}$ & YES & NO & YES & $\begin{array}{c}\text { YES } \\
\text { PARTIAL } \\
\end{array}$ & NO & NO & YES & YES & $\begin{array}{c}\text { YES } \\
\text { PARTIAL } \\
\end{array}$ & YES & $\mathrm{NA}$ & $\mathrm{NA}$ & NO & NO & $\mathrm{NA}$ & YES & Low \\
\hline Pal, 2013 [14] & YES & YES & YES & YES & YES & YES & YES & YES & YES & YES & YES & YES & YES & YES & YES & YES & High \\
\hline $\mathrm{Fu}, 2017$ [17] & YES & NO & YES & $\begin{array}{c}\text { YES } \\
\text { PARTIAL }\end{array}$ & NO & NO & $\begin{array}{c}\text { YES } \\
\text { PARTIAL }\end{array}$ & $\begin{array}{c}\text { YES } \\
\text { PARTIAL }\end{array}$ & NO & NO & NA & NA & NO & NO & NA & NO & Low \\
\hline Porter, 2016 [27] & YES & $\begin{array}{c}\text { YES } \\
\text { PARTIAL }\end{array}$ & YES & $\begin{array}{c}\text { YES } \\
\text { PARTIAL }\end{array}$ & YES & NO & YES & YES & $\begin{array}{c}\text { YES } \\
\text { PARTIAL }\end{array}$ & NO & NA & NA & YES & YES & NA & NO & Moderate \\
\hline $\begin{array}{l}\text { Anderson, } 2016 \\
{[37]}\end{array}$ & NO & NO & YES & $\begin{array}{c}\text { YES } \\
\text { PARTIAL } \\
\end{array}$ & NO & NO & $\begin{array}{c}\text { YES } \\
\text { PARTIAL } \\
\end{array}$ & $\begin{array}{c}\text { YES } \\
\text { PARTIAL }\end{array}$ & NO & NO & NA & NA & NO & NO & NA & YES & Very low \\
\hline Hou, 2016 [38] & YES & NO & YES & $\begin{array}{c}\text { YES } \\
\text { PARTIAL }\end{array}$ & YES & YES & YES & $\begin{array}{c}\text { YES } \\
\text { PARTIAL }\end{array}$ & $\begin{array}{c}\text { YES } \\
\text { PARTIAL }\end{array}$ & NO & YES & YES & NO & YES & YES & NO & Baja \\
\hline Cotter, 2014 [39] & NO & NO & NO & $\begin{array}{c}\text { YES } \\
\text { PARTIAL } \\
\end{array}$ & YES & YES & YES & YES & NO & YES & $\mathrm{NA}$ & $\mathrm{NA}$ & NO & YES & NA & YES & Very low \\
\hline David, 2016 [40] & NO & NO & YES & NO & NO & NO & YES & YES & NO & NO & $\mathrm{NA}$ & $\mathrm{NA}$ & NO & NO & $\mathrm{NA}$ & NO & Very low \\
\hline Bonoto, 2017 [41] & YES & NO & YES & YES & YES & YES & YES & YES & YES & NO & YES & YES & YES & YES & YES & YES & Moderate \\
\hline Drincic, 2016 [42] & YES & NO & YES & NO & NO & NO & NO & YES & NO & NO & NA & NA & NO & NO & NA & NO & Very low \\
\hline $\begin{array}{ll}\begin{array}{l}\text { El-Gayar, } 2013 \\
{[43]}\end{array} & \\
\end{array}$ & YES & NO & NO & $\begin{array}{c}\text { YES } \\
\text { PARTIAL } \\
\end{array}$ & YES & NO & YES & $\begin{array}{c}\text { YES } \\
\text { PARTIAL } \\
\end{array}$ & NO & NO & $\mathrm{NA}$ & $\mathrm{NA}$ & NO & NO & NA & NO & Very low \\
\hline Hood, 2016 [44] & NO & NO & NO & NO & NO & NO & NO & YES & NO & NO & NA & NA & NO & NO & NA & YES & Very low \\
\hline Veazie, 2018 [45] & YES & NO & YES & $\begin{array}{c}\text { YES } \\
\text { PARTIAL }\end{array}$ & YES & NO & YES & $\begin{array}{c}\text { YES } \\
\text { PARTIAL }\end{array}$ & $\begin{array}{c}\text { YES } \\
\text { PARTIAL }\end{array}$ & YES & $\mathrm{NA}$ & $\mathrm{NA}$ & YES & YES & NA & YES & Moderate \\
\hline $\begin{array}{ll}\text { Aminuddin, } 2019 \\
{[46]}\end{array}$ & NO & YES & NO & YES & YES & NO & NO & $\begin{array}{c}\text { YES } \\
\text { PARTIAL } \\
\end{array}$ & NO & YES & NO & NO & NO & NO & NO & YES & Very low \\
\hline Akbari, 2019 [47] & NO & NO & YES & $\begin{array}{c}\text { YES } \\
\text { PARTIAL } \\
\end{array}$ & YES & YES & $\begin{array}{c}\text { YES } \\
\text { PARTIAL } \\
\end{array}$ & $\begin{array}{c}\text { YES } \\
\text { PARTIAL } \\
\end{array}$ & YES & NO & YES & YES & YES & YES & YES & YES & Moderate \\
\hline Lunde, 2018 [48] & YES & $\begin{array}{c}\text { YES } \\
\text { PARTIAL } \\
\end{array}$ & YES & $\begin{array}{c}\text { YES } \\
\text { PARTIAL } \\
\end{array}$ & YES & YES & $\begin{array}{c}\text { YES } \\
\text { PARTIAL } \\
\end{array}$ & NO & $\begin{array}{c}\text { YES } \\
\text { PARTIAL } \\
\end{array}$ & NO & YES & YES & YES & YES & YES & YES & Moderate \\
\hline $\mathrm{Wu}, 2019$ [49] & NO & NO & YES & YES & YES & YES & $\begin{array}{c}\text { YES } \\
\text { PARTIAL }\end{array}$ & $\begin{array}{c}\text { YES } \\
\text { PARTIAL }\end{array}$ & $\begin{array}{c}\text { YES } \\
\text { PARTIAL }\end{array}$ & YES & YES & YES & YES & YES & YES & YES & Moderate \\
\hline
\end{tabular}

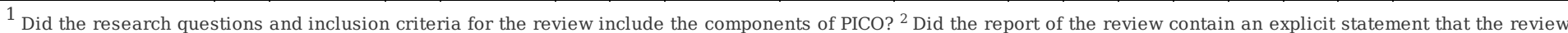

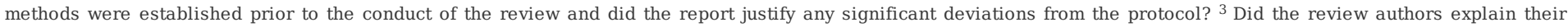

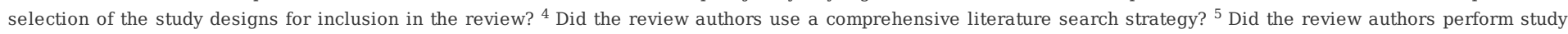

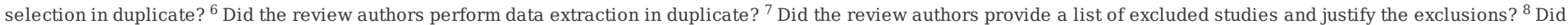

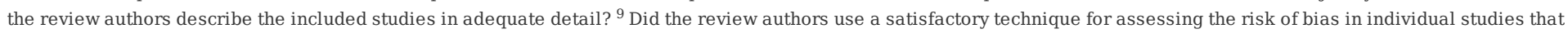

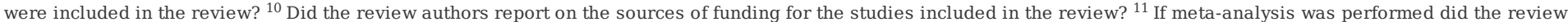

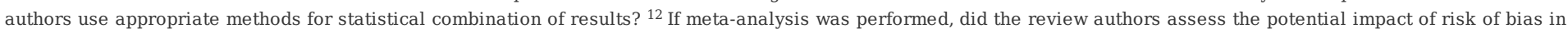

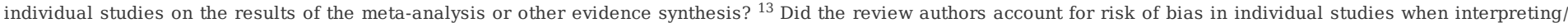

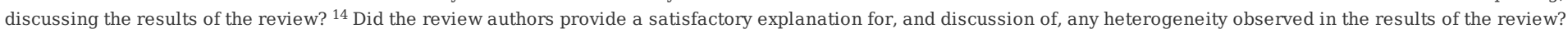

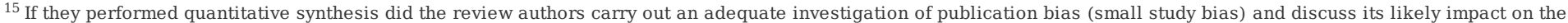
results of the review? ${ }^{16}$ Did the review authors report any potential sources of conflict of interest, including any funding they received for conducting the review?

NA $=$ Not applicable

\section{Characteristics and quality of the systematic reviews}

The main characteristics of the seven systematic reviews included in this review are shown in Table 2 . The complete analysis of their methodological quality is found in Table 1.

Although differences were observed in search methods, databases, inclusion and exclusion criteria, data extraction, quality assessments and statistical analyses, the seven systematic reviews generally provided an extensive description of the used methods, as well as the quality and general characteristics of the studies they included.

The studies included in the seven systematic reviews were conducted mainly in the United States, Asia and Europe. When we analyzed the original studies included in the reviews, we considered that 28 studies appeared in more than one systematic review.

The number of studies included in each analyzed review fell within the 8-19 range. In all cases, except for one study with a cross design featured in the review by Porter et al. [27], random clinical trials (RCTs) were included which assessed interventions based on using Apps to manage DM in adult patients. Interventions lasted between 1 and 12 months. The vast majority of the studies analyzed HbA1c as the primary outcome measure, which was also used to perform a meta-analysis in the reviews by Pal et al. [14], Bonoto et al. [41], Lunde et al. [48] and Wu et al. [49].

Table 2. General characteristics of the included systematic reviews 


\begin{tabular}{|c|c|c|c|c|c|c|c|c|c|}
\hline $\begin{array}{l}\text { First } \\
\text { author, } \\
\text { year / } \\
\text { source }\end{array}$ & $\begin{array}{l}\text { Search } \\
\text { strategy }\end{array}$ & $\begin{array}{l}\text { No. studies } \\
\text { included / } \\
\text { Total no. of } \\
\text { participants }\end{array}$ & Objective & Population & Intervention & Comparison & Outcome measures & $\begin{array}{l}\text { Time } \\
\text { (months) }\end{array}$ & Quality \\
\hline $\begin{array}{l}\text { Pal, } 2013 \\
14 \\
/ \\
\text { Cochrane } \\
\text { Database } \\
\text { of Syst } \\
\text { Rev }\end{array}$ & $\begin{array}{l}\text { Cochrane } \\
\text { Library, } \\
\text { MEDLINE, } \\
\text { EMBASE, } \\
\text { PsychINF, } \\
\text { Web of } \\
\text { Science, } \\
\text { CINAHL; + } \\
3 \text { databases } \\
\text { for thesis } \\
\text { Indicated } \\
\text { search } \\
\text { terms. } \\
\text { No } \\
\text { restrictions } \\
\text { with } \\
\text { publishing } \\
\text { date and } \\
\text { language. }\end{array}$ & $\begin{array}{l}16 \mathrm{RCT} \\
/ \\
3.578\end{array}$ & $\begin{array}{l}\text { Evaluate the effect } \\
\text { of DM self-control } \\
\text { interventions } \\
\text { based on computer } \\
\text { tools about health } \\
\text { status and CVRS. }\end{array}$ & $\begin{array}{l}\mathrm{DM} 2 \\
\geq 18 \\
\text { years }\end{array}$ & $\begin{array}{l}\text { Apps to help } \\
\text { patients acquire } \\
\text { knowledge, } \\
\text { skills (lifestyles) } \\
\text { and emotional } \\
\text { self-control } \\
\text { emotional } \\
\text { (handling } \\
\text { stress, anxiety } \\
\text { and social } \\
\text { support). }\end{array}$ & $\begin{array}{l}\text { Usual } \\
\text { healthcare }\end{array}$ & $\begin{array}{l}\text { Primary: } \\
\text { CVRS }(\mathrm{N}=5) \\
\text { HbA1c }(\mathrm{N}=16) \\
\text { Death by any cause } \\
(\mathrm{N}=2) \\
\text { Secondary: } \\
\text { Cognitive faculties: } \\
\text { knowledge/ } \\
\text { comprehension }(\mathrm{N}=4) \text {; } \\
\text { self-efficacy }(\mathrm{N}=2) \\
\text { Habits: physical } \\
\text { activity }(\mathrm{N}=5) \text {; diet } \\
\text { (N=6) } \\
\text { Social help }(\mathrm{N}=2) \\
\text { PAS and } \mathrm{PAD}(\mathrm{N}=5) \\
\text { Lipid profile }(\mathrm{N}=10) \\
\text { BMI/kg }(\mathrm{N}=5) \\
\text { Anxiety or depression } \\
\text { (N=6) } \\
\text { Complications }(\mathrm{N}=0) \\
\text { Adverse effects }(\mathrm{N}=1) \\
\end{array}$ & $1-12$ & High \\
\hline $\begin{array}{l}\text { Porter, } \\
2016^{27} \\
/ \\
\text { Nutrients }\end{array}$ & $\begin{array}{l}\text { Ovid } \\
\text { MEDLINE, } \\
\text { EMBASE, } \\
\text { CINAHL, } \\
\text { EBM } \\
\text { Reviews } \\
\text { Cochrane } \\
\text { Database of } \\
\text { Systematic } \\
\text { Reviews, } \\
\text { PsycINFO, } \\
\text { EBM } \\
\text { Reviews- } \\
\text { Health } \\
\text { Technology } \\
\text { Assessment. } \\
\\
\text { Indicated } \\
\text { search } \\
\text { terms. } \\
\text { No } \\
\text { restrictions } \\
\text { with } \\
\text { publishing } \\
\text { date and } \\
\text { language. }\end{array}$ & $\begin{array}{l}9(\mathrm{~N}=8 \mathrm{RCT} \\
+\mathrm{N}=1 \\
\text { Crossed }) \\
/ \\
1.234\end{array}$ & $\begin{array}{l}\text { Determine the } \\
\text { effect of using } \\
\text { Apps designed to } \\
\text { record the intake } \\
\text { of food or } \\
\text { nutrients on } \\
\text { controlling DM } \\
\text { and nutritional } \\
\text { outcomes. }\end{array}$ & $\begin{array}{l}\text { DM1 } \\
\text { DM2 } \\
\geq 18 \\
\text { years }\end{array}$ & $\begin{array}{l}\text { Apps for } \\
\text { recording food } \\
\text { intake, } \\
\text { glycemia, } \\
\text { physical activity } \\
\text { and medication. }\end{array}$ & $\begin{array}{l}\text { Usual } \\
\text { healthcare }\end{array}$ & $\begin{array}{l}\text { Primary: } \\
\text { HbA1c }(\mathrm{N}=9) \\
\text { Secondary: } \\
\text { Glycemia on an empty } \\
\text { stomach }(\mathrm{N}=4) \\
\text { BMI/kg/anthropometry } \\
(\mathrm{N}=6) \\
\text { Lipid profile }(\mathrm{N}=5) \\
\text { Diet }(\mathrm{N}=2) \\
\text { Satisfaction and } \\
\text { usefulness }(\mathrm{N}=4) \\
\text { Acceptance and } \\
\text { commitment }(\mathrm{N}=5)\end{array}$ & 3-12 & Moderate \\
\hline $\begin{array}{l}\text { Bonoto, } \\
2017^{41} \\
\text { / } \\
\text { JMIR } \\
\text { Mhealth } \\
\text { Uhealth }\end{array}$ & $\begin{array}{l}\text { MEDLINE, } \\
\text { Cochrane } \\
\text { Library } \\
\text { (CENTRAL), } \\
\text { LILACS. } \\
\\
\text { Indicated } \\
\text { search } \\
\text { terms. } \\
\text { Publishing } \\
\text { date 2008- } \\
2016 . \\
\text { No } \\
\text { language } \\
\text { restrictions. }\end{array}$ & $\begin{array}{l}13 \mathrm{RCT} \\
/ \\
1.263\end{array}$ & $\begin{array}{l}\text { Evaluate the } \\
\text { effectiveness of } \\
\text { Apps as a } \\
\text { healthcare } \\
\text { support. }\end{array}$ & $\begin{array}{l}\text { DM1 } \\
\text { DM2 } \\
\geq 18 \\
\text { years }\end{array}$ & $\begin{array}{l}\text { Apps that works } \\
\text { as an automatic } \\
\text { reminder to } \\
\text { help adhere to } \\
\text { DM self-control } \\
\text { measures } \\
\text { (glycemia, } \\
\text { weight, } \\
\text { medication, } \\
\text { food, physical } \\
\text { activity). }\end{array}$ & $\begin{array}{l}\text { Usual } \\
\text { healthcare }\end{array}$ & $\begin{array}{l}\text { Primary: } \\
\text { HbA1c }(\mathrm{N}=12) \\
\text { Hypogycemia episodes } \\
(\mathrm{N}=5) \\
\text { Secondary: } \\
\text { Glycemia on an empty } \\
\text { stomach }(\mathrm{N}=4) \\
\text { Body weight }(\mathrm{N}=6) \\
\text { SBP and DBP }(\mathrm{N}=4) \\
\text { Lipid profile }(\mathrm{N}=3) \\
\text { Quality of } \\
\text { life/satisfaction }(\mathrm{N}=6)\end{array}$ & $1-12$ & Moderate \\
\hline $\begin{array}{l}\text { Veazie, } \\
2018^{45} \\
\text { / } \\
\text { J Gen } \\
\text { Intern } \\
\text { Med }\end{array}$ & $\begin{array}{l}\text { Ovid } \\
\text { MEDLINE, } \\
\text { Cochrane } \\
\text { Database of } \\
\text { Systematic } \\
\text { Reviews. } \\
\\
\text { No } \\
\text { indicated } \\
\text { search } \\
\text { terms. } \\
\\
\text { Publishing } \\
\text { date } \\
\text { January } \\
\text { 2008-June } \\
\text { 2017. } \\
\\
\text { Restricted } \\
\text { to the } \\
\text { English } \\
\text { language. }\end{array}$ & $\begin{array}{l}15(\mathrm{~N}=13 \\
\mathrm{RCT}+\mathrm{N}=2 \\
\text { Subgroup } \\
\text { RCT }) \\
/ \\
\text { ne }\end{array}$ & $\begin{array}{l}\text { Examine the } \\
\text { characteristics, } \\
\text { clinical efficacy } \\
\text { and usability of } \\
\text { Apps for DM self- } \\
\text { management }\end{array}$ & $\begin{array}{l}\text { DM1 } \\
\text { DM2 } \\
\geq 18 \\
\text { years }\end{array}$ & $\begin{array}{l}\text { Apps to improve } \\
\text { diabetes self- } \\
\text { control (social } \\
\text { support, } \\
\text { education, } \\
\text { reminder of } \\
\text { activities, } \\
\text { recording } \\
\text { glycemia, } \\
\text { medication, } \\
\text { patient- } \\
\text { professional } \\
\text { communication). }\end{array}$ & $\begin{array}{l}\text { Usual } \\
\text { healthcare }\end{array}$ & $\begin{array}{l}\text { Primary: } \\
\text { HbA1c } \\
\text { Hypo/hyperglycemia } \\
\text { episodes } \\
\text { Glycemia on an empty } \\
\text { stomach } \\
\text { BP } \\
\text { Lipid profile } \\
\text { BMI/kg/anthropometry } \\
\text { Diabetic symptoms } \\
\text { Quality of life } \\
\text { Stress/depression } \\
\text { Satisfaction and } \\
\text { usefulness } \\
\text { Self-care and self- } \\
\text { efficacy }\end{array}$ & $2,5-12$ & Moderate \\
\hline
\end{tabular}




\begin{tabular}{|c|c|c|c|c|c|c|c|c|c|}
\hline $\begin{array}{l}\text { Akbari, } \\
2019^{47} \\
/ \\
\text { Diabetes } \\
\text { Metab } \\
\text { Syndr }\end{array}$ & $\begin{array}{l}\text { PubMed, } \\
\text { EMBASE, } \\
\text { Web of } \\
\text { Science, } \\
\text { Cochrane } \\
\text { Library } \\
\text { Databases. } \\
\text { Indicated } \\
\text { search } \\
\text { terms. } \\
\text { No } \\
\text { restrictions } \\
\text { with } \\
\text { publishing } \\
\text { date and } \\
\text { language. } \\
\\
\text { No } \\
\text { publishing } \\
\text { date } \\
\text { restrictions }\end{array}$ & $\begin{array}{l}9 \text { RCT } \\
/ \\
997\end{array}$ & $\begin{array}{l}\text { Evaluate the effect } \\
\text { of "m-health" on } \\
\text { the lipid profile of } \\
\text { patients with } \\
\text { metabolic } \\
\text { syndrome and } \\
\text { obesity, DM or } \\
\text { heart disease }\end{array}$ & $\begin{array}{l}\text { DM1 } \\
\text { DM2 } \\
\geq 18 \\
\geq 18 \\
\text { years }\end{array}$ & $\begin{array}{l}\text { Apps to improve } \\
\text { e lipid profile } \\
\text { through healthy } \\
\text { life habits } \\
\text { (physical } \\
\text { activity, food, } \\
\text { emotional } \\
\text { support, } \\
\text { medication, } \\
\text { smoking). }\end{array}$ & $\begin{array}{l}\text { Usual } \\
\text { healthcare }\end{array}$ & $\begin{array}{l}\text { Primary: } \\
\text { Lipìd profile }\end{array}$ & $3-12$ & Moderate \\
\hline $\begin{array}{l}\text { Lunde, } \\
2018^{48} \\
/ \\
\text { J Med } \\
\text { Internet } \\
\text { Res }\end{array}$ & $\begin{array}{l}\text { EMBASE, } \\
\text { MEDLINE, } \\
\text { CINAHL, } \\
\text { Academic } \\
\text { Research } \\
\text { Premier, } \\
\text { Cochrane } \\
\text { Reviews and } \\
\text { Trials. } \\
\text { Indicated } \\
\text { search } \\
\text { terms. } \\
\\
\text { Publishing } \\
\text { date before } \\
\text { 23/02/17. } \\
\\
\text { Restricted } \\
\text { to the } \\
\text { English } \\
\text { language. }\end{array}$ & $\begin{array}{l}8 \mathrm{RCT} \\
/ \\
1.201\end{array}$ & $\begin{array}{l}\text { Revise the } \\
\text { effectiveness of } \\
\text { interventionsbased } \\
\text { on Apps that } \\
\text { lasted a minimum } \\
\text { of } 3 \text { months to } \\
\text { promote changes } \\
\text { in lifestyles. }\end{array}$ & $\begin{array}{l}\text { DM1 } \\
\text { DM2 } \\
\geq 18 \text { years }\end{array}$ & $\begin{array}{l}\text { Apps to } \\
\text { monitor } \\
\text { glycemia, } \\
\text { BP and/or } \\
\text { diet habits }\end{array}$ & $\begin{array}{l}\text { Usual } \\
\text { healthcare }\end{array}$ & $\begin{array}{l}\text { Primary: } \\
\text { HbA1c }(\mathrm{N}=8) \\
\text { BMI/kg }(\mathrm{N}=6) \\
\text { Abdominal girth }(\mathrm{N}=3) \\
\text { Quality of life }(\mathrm{N}=3) \\
\text { Habits: physical activity } \\
(\mathrm{N}=1) \text {; diet }(\mathrm{N}=1)\end{array}$ & $3-12$ & Moderate \\
\hline $\begin{array}{l}\text { Wu, } 2019 \\
49 \\
\text { / } \\
\text { JMIR } \\
\text { Mhealth } \\
\text { Uhealth }\end{array}$ & $\begin{array}{l}\text { Cochrane } \\
\text { Central } \\
\text { Register of } \\
\text { Controlled } \\
\text { Trails in the } \\
\text { Cochrane } \\
\text { Library, } \\
\text { MEDLINE, } \\
\text { EMBASE, } \\
\text { CINAHL, } \\
\text { PsyscINFO. } \\
\\
\text { Indicated } \\
\text { search } \\
\text { terms. } \\
\text { Publishing } \\
\text { date } \\
01 / 01 / 06- \\
14 / 05 / 18 . \\
\\
\text { No } \\
\text { language } \\
\text { restrictions }\end{array}$ & $\begin{array}{l}19 \text { RCT } \\
/ 1.605\end{array}$ & $\begin{array}{l}\text { Summarize } \\
\text { existing clinical } \\
\text { evidence for the } \\
\text { efficiency of Apps } \\
\text { to modify lifestyles } \\
\text { in different DM } \\
\text { types. }\end{array}$ & $\begin{array}{l}\text { DM1 } \\
\text { DM2 } \\
\geq 18 \text { years }\end{array}$ & $\begin{array}{l}\text { Apps to help } \\
\text { to improve } \\
\text { lifestyles } \\
\text { (physical } \\
\text { activity, } \\
\text { food, } \\
\text { medication, } \\
\text { education). }\end{array}$ & $\begin{array}{l}\text { Usual } \\
\text { healthcare }\end{array}$ & $\begin{array}{l}\text { Primary: } \\
\text { HbA1c (N=16) } \\
\text { Secondary: } \\
\text { Lipid profile }(\mathrm{N}=7) \\
\text { SBP and DBP }(\mathrm{N}=10) \\
\text { Change in treatment } \\
(\mathrm{N}=3) \\
\text { Anxiety or depression } \\
(\mathrm{N}=4) \\
\text { BMI/kg }(\mathrm{N}=11) \\
\text { Quality of } \\
\text { life/satisfaction }(\mathrm{N}=5) \\
\text { Knowledge/self-care } \\
(\mathrm{N}=9) \\
\text { Abdominal girth }(\mathrm{N}=7) \\
\text { Habits: physical activity } \\
\text { (N=6); diet }(\mathrm{N}=2) \\
\text { Complications }(\mathrm{N}=1)\end{array}$ & $3-12$ & Moderate \\
\hline
\end{tabular}

No: Number / DM1: Type 1 Diabetes Mellitus / DM2: Type 2 Diabetes Mellitus 2 / ns: Not specified / RCT: random clinical trial / BP: Blood pressure / SDP: systolic blood pressure / DBP: diastolic blood pressure / BMI: body mass index / HbA1c: glycated hemoglobin / kg: Kilograms of body weight /HRQOL: health-related quality of life.

\section{Mobile applications and the lifestyles they address}

The identified mobile Apps and lifestyles are provided in detail in Table 3.

Twenty-three different apps were identified. The only App that was included in all seven systematic reviews was "BlueStar Diabetes", which offers free access for Apple and Android, and addresses food. We were unable to identify the commercial name of one App.

Regarding the lifestyles covered by Apps, eight included different questions about DM self-control in relation to food, five to physical activity, and 10 combined both these components. 
Most research into the effectiveness of Apps was conducted with individuals with DM2. Although their effectiveness for DM1 was also studied, no systematic reviews included studies about Gestational Diabetes (GD).

Table 3. The mobile applications included and the lifestyles they address

\begin{tabular}{|c|c|c|c|c|c|}
\hline App & Systematic Review & $\begin{array}{l}\text { Operating } \\
\text { system }\end{array}$ & DM type & Lifestyle & Cost \\
\hline Bees & Veazie, $2018^{45}$ & Apple/Android & DM1 & $\begin{array}{l}\text { Physical } \\
\text { activity and } \\
\text { food }\end{array}$ & Free \\
\hline BlueStar Diabetes & $\begin{array}{l}\text { Pal, } 2013^{14} / \text { Porter, } 2016^{27} \text { / Bonoto, } 2017^{41} \text { / Veazie, } 2018^{45} \text { / Akbari, } \\
2019^{47} \text { / Lunde, } 2018^{48} / \mathrm{Wu}, 2019^{49}\end{array}$ & Apple/Android & DM2 & Food & Free \\
\hline CollaboRhythm & Bonoto, $2017^{41}$ & ns & DM2 & $\begin{array}{l}\text { Physical } \\
\text { activity and } \\
\text { food }\end{array}$ & ns \\
\hline $\begin{array}{l}\text { Connected Wellness } \\
\text { Platform }\end{array}$ & Lunde, $2018^{48} / \mathrm{Wu}, 2019^{49}$ & Android & DM2 & $\begin{array}{l}\text { Physical } \\
\text { activity and } \\
\text { food }\end{array}$ & ns \\
\hline Diabeo Telesage & Bonoto, $2017^{41} /$ Veazie, $2018^{45} / \mathrm{Wu}, 2019^{49}$ & Apple/Android & DM1 & $\begin{array}{l}\text { Physical } \\
\text { activity and } \\
\text { food }\end{array}$ & Free \\
\hline Diabetes Diary & Veazie, $2018^{45} / \mathrm{Wu}, 2019^{49}$ & Apple/Android & DM1 & Food & Free \\
\hline Diabetes diet advisor & Pal, $2013^{14}$ & ns & DM2 & Food & ns \\
\hline $\begin{array}{l}\text { Diabetes Interactive } \\
\text { Diary (DID) }\end{array}$ & $\begin{array}{l}\text { Porter, } 2016^{27} \text { / Bonoto, } 2017^{41} \text { / Veazie, } 2018^{45} \text { / Akbari, } 2019^{47} / \mathrm{Wu} \text {, } \\
2019^{49}\end{array}$ & Android & DM1 & Food & Free \\
\hline Diabetes Pilot $^{\mathrm{TM}}$ & Porter, $2016^{27}$ & Apple & DM2 & Food & Payment \\
\hline $\begin{array}{l}\text { Diabetes Under } \\
\text { Control (DBEES) }\end{array}$ & Bonoto, $2017^{41}$ / Porter, $2016^{27}$ & Apple/Android & DM1 & $\begin{array}{l}\text { Physical } \\
\text { activity and } \\
\text { food }\end{array}$ & Free \\
\hline DialBetics & Porter, $2016^{27}$ / Akbari, $2019^{47}$ / Lunde, 201848 / Wu, 201949 & ns & DM2 & $\begin{array}{l}\text { Physical } \\
\text { activity and } \\
\text { food }\end{array}$ & ns \\
\hline eCoFit & Wu, $2019^{49}$ & Android & DM2 & $\begin{array}{l}\text { Physical } \\
\text { activity }\end{array}$ & Free \\
\hline $\begin{array}{l}\text { Few Touch } \\
\text { Application (FTA) }\end{array}$ & Bonoto, $2017^{41}$ / Porter, $2016^{27}$ / Lunde, $2018^{48}$ / Wu, $2019^{49}$ & ns & DM2 & $\begin{array}{l}\text { Physical } \\
\text { activity and } \\
\text { food }\end{array}$ & ns \\
\hline FoodLog & Porter, $2016^{27}$ / Akbari, $2019^{47}$ / Lunde, $2018^{48}$ / Wu, $2019^{49}$ & ns & DM2 & $\begin{array}{l}\text { Physical } \\
\text { activity and } \\
\text { food }\end{array}$ & ns \\
\hline Gather Health & Veazie, $2018^{45}$ & Apple/Android & DM2 & $\begin{array}{l}\text { Physical } \\
\text { activity and } \\
\text { food }\end{array}$ & Free \\
\hline Glucose Buddy & Bonoto, $2017^{41}$ / Veazie, $2018^{45} / \mathrm{Wu}, 2019^{49}$ & Apple/Android & DM1 & $\begin{array}{l}\text { Physical } \\
\text { activity and } \\
\text { food }\end{array}$ & Free \\
\hline LiFe! & Wu, $2019^{49}$ & Android & DM2 & $\begin{array}{l}\text { Physical } \\
\text { activity }\end{array}$ & Free \\
\hline mDiab & Veazie, $2018^{45}$ & Apple/Android & DM2 & $\begin{array}{l}\text { Physical } \\
\text { activity }\end{array}$ & Payment \\
\hline METADIETA & Bonoto, $2017^{41}$ & Apple/Android & DM2 & Food & Payment \\
\hline Monica & Bonoto, $2017^{41} /$ Lunde, $2018^{48} / \mathrm{Wu}, 2019^{49}$ & Android & DM2 & $\begin{array}{l}\text { Physical } \\
\text { activity }\end{array}$ & ns \\
\hline NICHE & Wu, $2019^{49}$ & ns & DM2 & $\begin{array}{l}\text { Physical } \\
\text { activity }\end{array}$ & Free \\
\hline WellTang & $\begin{array}{l}\text { Porter, } 2016^{27} \text { / Veazie, } 2018^{45} \text { / Akbari, } 2019^{47} \text { / Lunde, } 2018^{48} \text { / Wu, } \\
2019^{49}\end{array}$ & Apple & DM1/DM2 & Food & Free \\
\hline ne & Wu, $2019^{49}$ & ns & DM2 & Food & ns \\
\hline
\end{tabular}

App: Mobile Aplication / DM1: Type 1 Diabetes Mellitus / DM2: Type 2 Diabetes / ns: Not specified

\section{Primary outcome measures}

Pal et al. [14] performed a meta-analysis of the 11 studies, which provided sufficient data on HbA1c levels. They found statistically significant differences between the results of the intervention groups and the control groups, with lower $\mathrm{HbA} 1 \mathrm{c}$ values in the intervention subjects. The impact of the intervention was significantly stronger $(P<.001)$ in the three studies that used mobile phones, with an effect on $\mathrm{HbA} 1 \mathrm{c}$ of $-5,5$ $\mathrm{mmol} / \mathrm{mol}$ or $-0.5 \%\left(95 \% \mathrm{Cl}:-0.74\right.$ to $-0.26 ; P=.59 ;\left.\right|^{2}=0 \%$ ). However, the effects of interventions seemed to disappear with time as the analysis of the results of the studies with a duration equal to or longer than 6 months was not statistically significant.

In four of the nine studies they analyzed, Porter et al. [27] found a statistically significant improvement in HbA1c of diabetics in the intervention group compared to the control group, while the other studies observed no significant differences between the two groups. 
In the review by Bonoto et al. [41], in six of the 12 studies that included HbA1c values, a statistically significant difference was found in the reduction of this parameter in favor of intervention groups. Likewise, the meta-analysis carried out in this review showed the effectiveness of using mobile applications to control diabetes, with an average difference of $-0.44 \%\left(95 \% \mathrm{Cl}\right.$ : 0.59 to $\left.-0.29 ; P<.10 ; I^{2}=32 \%\right)$, which was statistically significant $(P<.001)$.

Veazie et al. [45] identified two Apps to control DM1 (Glucose Buddy and Diabeo Telesage) and three others for DM2 (Gather Health, BlueStar and WellTang), whose use demonstrated better statistics and clinical significance for HbA1c than the control groups (CG).

Lunde et al. [48] included three studies to evaluate the effectiveness of short-term apps, and four long-term studies. The result was a significant decrease in $\mathrm{HbA} 1 \mathrm{c}$ in both cases, although the quality of the evidence was poor for the short-term and moderate for the long-term Apps.

However, Wu et al. [49] did not reveal any significant difference in $\mathrm{HbA} 1 \mathrm{c}$ between the intervention group and control group with DM1, in both the short $-0.09 \%$ (95\%Cl: 0.34 to $\left.-0.15 ; P=.18 ; I^{2}=39 \%\right)$ and long term $-0.21 \%\left(95 \% \mathrm{Cl}-0.52\right.$ a $\left.-0.09 ; P=.17 ; I^{2}=64 \%\right)$ terms. In the Apps that addressed patients with $\mathrm{DM} 2$, a statistically significant difference appeared in both the short and long terms with $0.35 \%(95 \% \mathrm{Cl}:-0.48$ to -0.21 ; $P<0.01$; $\left.\mathrm{I}^{2}=0 \%\right)$.

To assess the effect of interventions based on using Apps on diabetic patients' healthy lifestyles, we also analyzed changes in body weight or $\mathrm{BMI}$ as a result of the intervention. Lunde et al. [48], included three studies in which the IG reduced weight significantly [50-52]. In the review by Pal et al. [14], only two of the five studies using mobile devices in their interventions reported changes in body weight and BMI [51,54]. However, these authors found no significant differences between the intervention groups and control groups. Bonoto et al. [41] did not observe any significant differences in their combined analysis of the results of four studies with data on changes in participants' body weight [mean difference: -0.39 (95\% Cl: -1.43 to $\left.0.66 ; P=.47 ; I^{2}=0 \%\right)$ ]. Similarly in their review, Porter et al. [27] described that none of the six studies with body weight or BMI data found significant changes linked with the intervention. Only one study indicated a slight reduction in the BMI of all the groups, but did not provide any data [53]. Finally, none of the participants in the studies analyzed by Veazie et al. [45] reported improvements in body weight or BMI.

\section{Secondary Outcome Measures}

Health-related quality of life (HRQL) was analyzed as a secondary outcome measure of interventions based on the use of Apps. Of the 16 studies included in the review by Pal et al. [14], five provided results on HRQL. However, these authors did not observe any significant improvement in the HRQL of the intervention subjects compared to the control subjects. Bonoto et al. [41] found that three studies yielded positive and statistically significant changes in both the quality of life of and satisfaction with the treatment of the patients in the intervention group. The improvements reported by the participants using Apps included perceiving hyperglycemia episodes, social relationships, feeling less fear of hypoglycemia and that Apps helped them to control their treatment and to maintain healthier dietary habits [54-56]. Conversely, Veazie et al. [45] found no evidence for improvement in the quality of life of the diabetic patients who used mobile Apps.

Some of the studies included in the seven analyzed reviews provided results on the lipid profile of diabetic subjects, which may be related to changes in their dietary habits and lifestyles. In connection with this, Pal et al. [14] carried out a meta-analysis with data from seven studies and found that the effect of the intervention on the participants' lipid profile was not significant $(P=.17)$, with a mean difference to the control group of -0.11 (95\%Cl:-0.28 to $\left.0.05 ; P=.03 ; I^{2}=57 \%\right)$. A significant improvement in the lipid profile was observed in only one intervention study based on using mobile devices, specifically a reduction in TC, LDL-C and TG levels. Bonoto et al. [41] and Akbari et al. [47] also found no significant differences in their meta-analysis of TC, HDL-C, LDL-C and TG levels. Porter et al. [27] and Veazie et al. [45] identified one study which observed a significant lowering $(P=.04)$ of triglyceride levels, but not the remaining lipids, in the participants who used a mobile app to control DM1 (Diabetes Interactive Diary) [55].

Regarding changes in diabetic patients' blood pressure, one study included in the review by Pal et al. [14] found a statistically significant decrease in SBP $(127 \pm 14 \mathrm{mmHg}$ to $120 \pm 19 \mathrm{mmHg} ; P=.001)$ and DBP $(78 \pm 10 \mathrm{mmHg}$ to $74 \pm 8 \mathrm{~mm} \mathrm{Hg}, P<.001)$ in the intervention group [57]. Bonoto et al. [41] did not observe any significant differences for the intervention and control groups of four studies in participants' SBP and DBP. Similarly, no intervention analyzed in the review by Veazie et al. [45] revealed participants' improved blood pressure.

Finally in a combined analysis of fasting blood glucose results from four studies, Bonoto et al. [41] reported no significant differences between the intervention and control groups. In the review by Porter et al. [27], two studies described a significantly more marked reduction ( $P<.01)$ in fasting blood glucose levels for the intervention group than for the control group [56,59], while two other studies did not observe any significant differences between these two groups [56,58]. Veazie et al. [45] identified one app (WellTang), whose use for DM2 demonstrated better fasting blood glucose values than for the control subjects.

\section{Adverse effects of interventions}


One important aspect to bear in mind when assessing the efficiency of Apps designed to improve DM self-control is that adverse effects may appear.

Of all the studies included in the seven reviews analyzed herein, a few describe adverse reactions reported by the participants of the intervention groups. Pal et al. [14] found a non significant increase in the frequency of mild hypoglycemia episodes in the intervention group, with no differences in severe or nocturnal hypoglycemia episodes [54]. Five studies included in the review by Bonoto et al. [41] reported hypoglycemia episodes. One reports averages of 30 and 33 mild episodes in the the intervention and the control group, respectively, and a severe episode in the control group [56]. In three other studies, no significant differences were observed between groups [55,60,61]. In a fifth study, the relative risk of severe hypoglycemia episodes was lower in IG (0:14; 95\% Cl: 0.07-0.029) [54]. One of the studies in the review by Wu et al. [49] found no statistically significant differences between the hypoglycemic events of IG and CG [56]. Two mobile apps (Diabetes Diary and Diabetes Interactive Diary) reviewed by Veazie et al. [45] showed improvements in hypoglycemic episodes of DM1.

Regarding adverse psychological effects, such as stress, anxiety or depression, Wu et al. [49] described a significant improvement in anxiety and depression symptoms in four studies [52,62-64].

\section{Discussion}

This work aimed to provide a broad view of the research conducted about using mobile Apps that address lifestyles to control and manage DM. As far as we know, this is the first review of systematic reviews (umbrella review) that considers that the effect of Apps on improving the metabolic control of DM patients.

Although many systematic reviews were found by the literature search, very few met sufficient quality criteria to be included in the present work. Given the variability of Apps and outcome measures, a decision was made to not include the data of the original studies in a meta-analysis.

The seven systematic reviews that acted as the basis for our study showed a clear benefit of mobile Apps that deal with lifestyles to improve DM patients' short-term glycemic control. These data need to be interpreted cautiously because other reasons could have intervened in HbA1c lowering, such as the persuasion of Apps to modify lifestyles [49] or health professionals' access via Apps that include remote communication tools [41]. Indeed the results of a recent pragmatic multicenter clinical random controlled trial did not indicate any differences between the IG (intervention group) and the CG for the primary clinical outcome of glycemic control measured by HbA1c, which also occurred in secondary outcomes like quality of life and behaviors stemming from medical healthcare uses [65].

Finding a convincing explanation for this phenomenon might be a complex matter. Our findings coincide with those of other authors who stated that most studies did not take into account the basis of behavioral health theories when developing Apps [66].

The scientific literature about behavioral health models in which Apps participate reveal very little discussion about behavioral health theories or models that provide a basis on which to support intervention [67]. One possible convincing explanation could lie in the theory of controlling interventions based on other theories. This theory postulates that a synergic association exists between receiving information about someone's behavior (via "self-control" or feedback") and obtaining a strategy with which to act on this information ("planning action" or "information as to how and where to perform behavior"). The former provides a sign and/or motivation for the latter.

The review by Pal et al. [14] does not provide sufficient evidence with which interventions improve cardiovascular risk factors (blood pressure, lipid profile and body weight) or cognitive, behavioral or emotional outcomes.

Our findings about long-term effects coincide with those published by other authors by identifying only one positive short-term effect on HbA1c. Indeed very few studies have found a positive long-term effect of Apps on $\mathrm{HbA1c}$ [68].

In the subgroups analysis, modification of lifestyles would have a stronger effect on DM2 patients than DM1 patients. This could be explained by the DM1 control depending largely on questions about administering insulin and not about amending lifestyles, which are the main cause for DM2 to appear [6]. Wu et al. consider that to ensure Apps being very efficient, a specific design is necessary for all DM subtypes [49]. This must also be done extensively for GD.

Using Apps to control DM seems to reinforce the self-control perception by providing DM patients with better health information and education. It can also increase patient security as to how to deal with their disease by mainly reducing their fear of not knowing how to treat possible hypoglycemia episodes [49,58], and to improve their quality of life [41]. Nonetheless, the impact of these Apps on long-term outcomes, such as quality of life, high blood pressure or frequent diabetes complications (neuropathy and retinopathy), remain unclear. More rigorous longer terms studies are still required to carefully consider the potential of the interaction between patients and health professionals/study personnel (Veazie et al. [45] and Lunde et al. [48]), along with their effects on DM-related mortality [69]. 
All the studied Apps focused on two lifestyles, which are most important to control DM well: food and physical exercise. This makes sense as both questions are dealt with simultaneously in clinical practice because they are closely related [6]. However, no Apps that addressed other lifestyles with a high prevalence, like smoking habit, were included [70].

Apart from the characteristics that are typical of Apps to deal with food and physical exercise, others were described because they appear to contribute to improve DM patients' glycemic control to a great extent, such as being able to store and feedback data about blood glucose, support to control doses and therapy with medicines being met and, finally, access to communicate with health professionals.

Among all the studied works, very few described the adverse effects of using Apps. It is necessary to bear in mind that despite potential benefits for patients, the Apps used to calculate insulin doses imply the risk of incorrect dose recommendations that range from those that lead to suboptimum disease control to potentially lethal consequences [71].

Indeed until quite recently, the growth and early adoption of technology both tend to lie mainly in healthcare suppliers' hands. Nowadays, the digital era has extended patients' access to technology. Most of the population has access to portable devices, mobile Apps, with better access to electronic medical records and health data over the Internet. The main leading role of technology in health management, and greater user accessibility and autonomy, are changing the patient's position from a passive person receiving health care to someone who also participates in managing his/her health.

\section{Limitations}

Some limitations appear to interpret and extrapolate the findings of this review. The few data that cover a period longer than 12 months is a major limitation if we consider the chronic condition of DM, and many of its complications are manifiested after the disease has been longstanding. Further evidence is necessary to contemplate studies that include longer follow-up peridos.

Evaluating the quality of the studied basic reviews was done using AMSTAR for its clear validity and reliability, which helps to identify the best quality evidence for each outcome without prejudicing the quality of the original studies that acted as the basis of the studied reviews. Given this scale's subjectivity component, the evaluation was done both step-wise and independently to minimize this risk.

Another important limitation arose when comparing studies as a result of the low clinical homogeneity of the seven included systematic reviews. Differences were observed in the literature search methods, the inclusion and exclusion criteria, the evaluation of the quality of individual studies, the extraction of primary and secondary outcome measures, and the analysis of the results. The meta-analyses included in five reviews analyzed only a few outcome measures.

\section{Conclusions}

The outcomes of this review will support the use of Apps to improve short-term glycemic control in DM patients. All the examined Apps centered on dealing with food and physical exercise. No signicifant adverse effects were identified for users of Apps.

Apps' beneficial long-term effect on health for diabetic patients was much weaker. Therefore, more in-depth research is necessary as far as the design, features and effectiveness of studied mobile Apps are concerned to encourage healthy lifestlyes for DM patients.

\section{Abbreviations}

SERGAS: the Galician Health Service

HRQOF: health-related quality of life

ECA: controlled random clinical trial

ns: not specified

HbA1c: glycated hemoglobin

BMI: body mass index

SBP: systolic blood pressure

DBP: diastolic blood pressure

TC: total cholesterol 
C-LDL: low-density lipoprotein cholesterol

C-HDL: high-desnity lipoprotein cholesterol

TG: triglycerides

APPs: mobile applications

WHO: World Health Organization

IDF: International Diabetes Federation

DM: diabetes mellitus

DM1: Type 1 diabetes mellitus

DM2: Type 2 diabetes mellitus

GD: gestational diabetes

eHealth: electronic health

PRISMA: Preferred Reporting Items for Systematic reviews and Meta-Analyses

PROSPERO: International prospective register of systematic reviews

NDLTD: Networked Digital Library of Theses and Dissertations

AMSTAR: A Measurement Tool to Assess Systematic Reviews-2

IG: intervention group

CG: control group

\section{Declarations}

\section{Author contributions}

All the authors were involved in drafting the manuscript. All the authors contributed to develop the selection criteria, the bias risk assessment strategy and the data extraction criteria. AAMQ developed the search strategy. ACF provided her experience in technology assessment. FJRC provided his experience in research into DM. All the authors read, provided comments on and approved the final manuscript.

\section{Conflicts of interest}

None declared.

\section{References}

1. International Diabetes Federation. Diabetes Atlas de la FID. Octava edi. Suvi Karuranga, Joao da Rocha Fernandes, Yadi Huang BM, editor. 8, Federación Internacional de Diabetes. 2017. p. 0-148. ISBN: 9782930229812

2. Forouzanfar MH, Alexander L, Anderson HR, Bachman VF, Biryukov S, Brauer M, et al. Global, regional, and national comparative risk assessment of 79 behavioural, environmental and occupational, and metabolic risks or clusters of risks in 188 countries, 1990-2013: a systematic analysis for the Global Burden of Disease Study 2013. Lancet. 2015 Dec 5;386(10010):2287-323. PMID: 26364544

3. Ley SH, Hamdy O, Mohan V, Hu FB. Prevention and management of type 2 diabetes: dietary components and nutritional strategies. Lancet. 2014;383(9933):1999- PMID: 24910231

4. GBD 2015 Disease and Injury Incidence and Prevalence Collaborators T, Allen C, Arora M, Barber RM, Bhutta ZA, Brown A, et al. Global, regional, and national incidence, prevalence, and years lived with disability for 310 diseases and injuries, 1990-2015: a systematic analysis for the Global Burden of Disease Study 2015. Lancet. 2016 Oct 8;388(10053):1659-724. PMID: 27733284

5. Lewis TL, Boissaud-Cooke MA, Aungst TD, Eysenbach G. Consensus on Use of the Term “App” Versus "Application” for Reporting of mHealth Research. J Med Internet Res. 2014 Jul 17;16(7):e174. PMID: 25033233 
6. American Diabetes Association. Standards of Medical Care in Diabetes 2020. Diabetes Care. 2020 Jan;43(1):S1-S212

7. World Health Organization. mHealth: New horizons for health through mobile technologies. In: Global Observatory for eHealth series. Switzerland: 2011; 2011. p. 102. ISBN 9789241564250

8. Rehman H, Kamal AK, Sayani S, Morris PB, Merchant AT, Virani SS. Using Mobile Health (mHealth) Technology in the Management of Diabetes Mellitus, Physical Inactivity, and Smoking. Curr Atheroscler Rep. 2017;19(4):16. PMID: 28243807

9. Chomutare T, Fernandez-Luque L, Arsand E, Hartvigsen G. Features of mobile diabetes applications: review of the literature and analysis of current applications compared against evidence-based guidelines. J Med Internet Res. 2011;13(3):e65. PMID: 21979293

10. Muralidharan S, Ranjani H, Anjana R, Allender S, Mohan V. Mobile health technology in the prevention and management of Type 2 Diabetes. Indian J Endocrinol Metab. 2017. 21(2):334. PMID: 28459035

11. Whitehead L, Seaton P. The Effectiveness of Self-Management Mobile Phone and Tablet Apps in Long-term Condition Management: A Systematic Review. J Med Internet Res. 2016;18(5):e97. PMID: 27185295

12. Heitkemper EM, Mamykina L, Travers J, Smaldone A. Do health information technology self-management interventions improve glycemic control in medically underserved adults with diabetes? A systematic review and meta-analysis. J Am Med Inform Assoc. 2017;24(5):1024PMID: 28379397

13. McMillan KA, Kirk A, Hewitt A, MacRury S. A Systematic and Integrated Review of Mobile-Based Technology to Promote Active Lifestyles in People With Type 2 Diabetes. J Diabetes Sci Technol. 2017;11(2):299-PMID: 27334301

14. Pal K, Eastwood SV, Michie S, et al. Computer-based diabetes self-management interventions for adults with type 2 diabetes mellitus. Cochrane Database Syst Rev. 2013;2013(3):CD008776. PMID: 23543567

15. Changizi M, Kaveh MH. Effectiveness of the mHealth technology in improvement of healthy behaviors in an elderly population-a systematic review. Mhealth. 2017;3:51. PMID: 29430455

16. Grock S, Ku JH, Kim J, Moin T. A Review of Technology-Assisted Interventions for Diabetes Prevention. Curr Diab Rep. 2017;17(11):107. PMID: 28942537

17. Fu H, McMahon SK, Gross CR, Adam TJ, Wyman JF. Usability and clinical efficacy of diabetes mobile applications for adults with type 2 diabetes: A systematic review. Diabetes Res Clin Pract. 2017;131:70- PMID: 28692830

18. Rossi MG, Bigi S. mHealth for Diabetes support: a systematic review of Apps available on the Italian market. Mhealth. 2017;3:16-16. PMID: 28567412

19. Wang Y, Xue H, Huang Y, Huang L, Zhang D. A Systematic Review of Application and Effectiveness of mHealth Interventions for Obesity and Diabetes Treatment and Self-Management. Adv Nutr An Int Rev J. 2017 May 15;8(3):449-62. PMID: 28507010

20. Joiner KL, Nam S, Whittemore R. Lifestyle interventions based on the diabetes prevention program delivered via eHealth: A systematic review and meta-analysis. Prev Med. 2017;100:194- PMID: 28456513

21. S, Jiang H, Luo Y, Zhang D. Application of diabetes phone recipe software in diet intervention for patients with type 2 diabetes. Chinese Nurs Res 2017;31(11):1407-1408. DOI: 10.3969/j.issn.1009-6493.2017.11.042

22. Cui M, Wu X, Mao J, Wang X, Nie M. T2DM self-management via smartphone Applications: A systematic review and meta-analysis. Barengo NC, editor. PLoS 2016 Nov 18;11(11):e0166718. PMID: 27861583

23. Hartz J, Yingling L, Powell-Wiley TM. Use of Mobile Health Technology in the Prevention and Management of Diabetes Mellitus. Curr Cardiol Rep. 2016;18(12):130. PMID: 27826901

24. Müller AM, Alley S, Schoeppe S, Vandelanotte C. The effectiveness of e-\& mHealth interventions to promote physical activity and healthy diets in developing countries: A systematic review. Int J Behav Nutr Phys Act. 2016;13(1):109. PMID: 27724911

25. Garabedian LF, Ross-Degnan D, Wharam JF. Mobile Phone and Smartphone Technologies for Diabetes Care and Self-Management. Curr Diab Rep. 2015;15(12):109. PMID: 26458380

26. Marcolino MS, Oliveira JAQ, D’Agostino M, Ribeiro AL, Alkmim MBM, Novillo- Ortiz D. The Impact of mHealth Interventions: Systematic Review of Systematic Reviews. JMIRMhealthUhealth. 2018 Jan 17;6(1):e23. PMID: 29343463

27. Porter J, Huggins CE, Truby H, Collins J. The Effect of Using Mobile Technology-Based Methods That Record Food or Nutrient Intake on Diabetes Control and Nutrition Outcomes: A Systematic Review. Nutrients. 2016;8(12):815. PMID: 27999302

28. O'Connor S, Hanlon P, O'Donnell CA, Garcia S, Glanville J, Mair FS. Barriers and facilitators to patient and public engagement and recruitment to digital health interventions: protocol of a systematic review of qualitative studies. BMJ Open. 2016;6(9):e010895. PMID: 27591017

29. European Commission. Green Paper on Mobile Health in the EU. Brussels: European Commission; 2014 . p.20

30. Powers MA, Bardsley J, Cypress M, Duker P, Funnell MM, Fischl AH, et Diabetes Self-management Education and Support in Type 2 Diabetes: A Joint Position Statement of the American Diabetes Association, the American Association of Diabetes Educators, and the Academy of Nutrition and Dietetics. Diabetes Educ. 2015 Aug 5;41(4):417-30. PMID: 26047627

Page $13 / 17$ 
31. Aromataris E, Fernandez R, Godfrey CM, Holly C, Khalil H, Tungpunkom P. Summarizing systematic reviews: methodological development, conduct and reporting of an umbrella review Approach. Int J Evid Based Healthc. 2015;13(3):132-140. PMID: 26360830

32. Higgins JPT, Green S (Editors). Cochrane Handbook for Systematic Reviews of Interventions Version 5.1.0. The Cochrane Collaboration, March 2011

33. Aromataris E, Munn Z (Editors). Joanna Briggs Institute Reviewer's Manual. The Joanna Briggs Institute, 2017.

34. Moher D, Liberati A, Tetzlaff J, Altman DG; PRISMA Group. Preferred reporting items for systematic reviews and meta-analyses: the PRISMA statement. Int J Surg. 2010;8(5):336- PMID: 20171303

35. hea BJ, Reeves BC, Wells G, et al. AMSTAR 2: a critical appraisal tool for systematic reviews that include randomised or non-randomised studies of healthcare interventions, or both. BMJ. 2017;358:j4008. PMID: 28935701

36. Represas-Carrera FJ, Martínez-Qués AA, Clavería-Fontán A. Effectiveness of mobile Applications on healthy lifestyles aimed at diabetic patients: review of systematic reviews. PROSPERO 2019 CRD42019133685.

37. Anderson K, Emmerton LM. Contribution of mobile health applications to self-management by consumers: review of published evidence. Aust Health Rev. 2016;40(5):591- PMID: 26681206

38. Hou C, Carter B, Hewitt J, Francisa T, Mayor S. Do Mobile Phone Applications Improve Glycemic Control (HbA1c) in the Self-management of Diabetes? A Systematic Review, Meta-analysis, and GRADE of 14 Randomized Trials. Diabetes Care. 2016;39(11):2089- PMID: 27926892

39. Cotter AP, Durant N, Agne AA, Cherrington AL. Internet interventions to support lifestyle modification for diabetes management: a systematic review of the evidence. J Diabetes Complications. 2014;28(2):243- PMID: 24332469

40. David SK, Rafiullah MR. Innovative health informatics as an effective modern strategy in diabetes management: a critical review. Int J Clin Pract. 2016;70(6):434- PMID: 27238962

41. Bonoto BC, de Araújo VE, Godói IP, de Lemos LL, Godman B, Bennie M, Diniz LM, Junior AA. Efficacy of Mobile Apps to Support the Care of Patients With Diabetes Mellitus: A Systematic Review and Meta-Analysis of Randomized Controlled Trials. JMIR Mhealth Uhealth. 2017;5(3):e4. PMID: 28249834

42. Drincic A, Prahalad P, Greenwood D, Klonoff DC. Evidence-based Mobile Medical Applications in Diabetes. Endocrinol Metab Clin North Am. 2016;45(4):943- PMID: 27823614

43. El-Gayar O, Timsina P, Nawar N, Eid W. Mobile applications for diabetes self-management: status and potential. J Diabetes Sci Technol. 2013;7(1):247- PMID: 23439183

44. Hood M, Wilson R, Corsica J, Bradley L, Chirinos D, Vivo A. What do we know about mobile Applications for Diabetes self-management? A review of reviews. J Behav Med. 2016 Dec 13;39(6):981-94. PMID: 27412774

45. Veazie S, Winchell K, Gilbert J, Paynter R, Ivlev I, Eden KB, Nussbaum K, Weiskopf N, Guise JM, Helfand M. Rapid Evidence Review of Mobile Applications for Self-management of Diabetes. J Gen Intern Med. 2018;33(7):1167-1176. PMID: 29740786

46. Aminuddin HB, Jiao N, Jiang Y, Hong J, Wang W. Effectiveness of smartphone-based self-management interventions on self-efficacy, selfcare activities, health-related quality of life and clinical outcomes in patients with type 2 diabetes: A systematic review and meta-analysis. Int J Nurs Stud. 2019;103286. PMID: 30827741

47. Akbari M, Lankarani KB, Naghibzadeh-Tahami A, et al. The effects of mobile health interventions on lipid profiles among patients with metabolic syndrome and related disorders: A systematic review and meta-analysis of randomized controlled trials. Diabetes Metab Syndr. 2019;13(3):1949- PMID: 31235120

48. Lunde P, Nilsson BB, Bergland A, Kværner KJ, Bye A. The Effectiveness of Smartphone Apps for Lifestyle Improvement in Noncommunicable Diseases: Systematic Review and Meta-Analyses. J Med Internet Res. 2018;20(5):e162. PMID: 29728346

49. Wu X, Guo X, Zhang Z. The Efficacy of Mobile Phone Apps for Lifestyle Modification in Diabetes: Systematic Review and Meta-Analysis. JMIR Mhealth Uhealth. 2019;7(1):e12297. PMID: 30664494

50. Karhula T, Vuorinen AL, Rääpysjärvi K, et al. Telemonitoring and Mobile Phone-Based Health Coaching Among Finnish Diabetic and Heart Disease Patients: Randomized Controlled Trial. J Med Internet Res. 2015;17(6):e153. PMID: 26084979

51. Orsama AL, Lähteenmäki J, Harno K, et al. Active assistance technology reduces glycosylated hemoglobin and weight in individuals with type 2 diabetes: results of a theory-based randomized trial. Diabetes Technol Ther. 2013;15(8):662- PMID: 23844570

52. Wayne N, Perez D, Kaplan D, Ritvo P. Health coaching reduces HbA1c in type 2 diabetic patients from a lower-socioeconomic status community: a randomized controlled trial. J Med Internet Res 2015;17(10):e224. PMID: 26441467

53. Forjuoh SN, Bolin JN, Huber JC Jr, et al. Behavioral and technological interventions targeting glycemic control in a racially/ethnically diverse population: a randomized controlled trial. BMC Public Health. 2014;14:71. PMID: 24450992

54. Rossi MC, Nicolucci A, Lucisano G, Pellegrini F, Di Bartolo P, Miselli V, Anichini R, Vespasiani G; Did Study Group. Impact of the "Diabetes Interactive Diary" telemedicine system on metabolic control, risk of hypoglycemia, and quality of life: a randomized clinical trial in type 1 Diabetes. Diabetes Technol Ther. 2013 Aug;15(8):670-679. PMID: 23844569

Page $14 / 17$ 
55. Rossi MC, Nicolucci A, Di Bartolo P, Bruttomesso D, Girelli A, Ampudia FJ, Kerr D, Ceriello A, Mayor Cde L, Pellegrini F, Horwitz D, Vespasiani G. Diabetes Interactive Diary: a new telemedicine system enabling flexible diet and insulin therapy while improving quality of life: an openlabel, international, multicenter, randomized study. Diabetes Care. 2010 Jan;33(1):109-115. PMID: 19808926

56. Berndt R, Takenga C, Preik P, Kuehn S, Berndt L, Mayer H, et al. Impact of information technology on the therapy of type-1 Diabetes: a case study of children and adolescents in Germany. J Pers Med. 2014;4(2):200-217. PMID: 25563223

57. Yoo HJ, Park MS, Kim TN, Yang SJ, Cho GJ, Hwng TG, Baik SH, Choi DS, Park GH, Choi KM. A ubiquitous chronic disease care system using cellular phones and the internet. Diabet Med. 2009;26(6):628-635. PMID: 19538239

58. Zhou W, Chen M, Yuan J, Sun Y. Welltang - A smart phone-based Diabetes management Application - Improves blood glucose control in Chinese people with Diabetes. Diabetes Res Clin Pract. 2016 Jun;116:105-110. PMID: 27321324

59. Waki K, Fujita H, Uchimura Y, Omae K, Aramaki E, Kato S, Lee H, Kobayashi H, Kadowaki T, Ohe K. DialBetics: A Novel Smartphone-based Self-management Support System for Type 2 Diabetes Patients. J Diabetes Sci Technol. 2014 Mar;8(2):209-215. PMID: 24876569

60. Hsu WC, Lau KH, Huang R, Ghiloni S, Le H, Gilroy S, et al. Utilization of a Cloud-Based Diabetes Management Program for Insulin Initiation and Titration Enables Collaborative Decision Making Between Healthcare Providers and Patients. Diabetes Technol Ther. 2016 Feb;18(2):59-67. PMID: 26645932

61. Charpentier G, Benhamou P, Dardari D, Clergeot A, Franc S, Schaepelynck-Belicar P, et al. The Diabeo software enabling individualized insulin dose adjustments combined with telemedicine support improves $\mathrm{HbA} 1 \mathrm{c}$ in poorly controlled type 1 diabetic patients: a 6-month, randomized, open-label, parallel-group, multicenter trial (TeleDiab 1 Study). Diabetes Care. 2011 Mar;34(3):533-539. PMID: 21266648

62. Holmen H, Torbjørnsen A, Wahl AK, Jenum AK, Småstuen MC, Arsand E, Ribu L. A Mobile Health Intervention for Self-Management and Lifestyle Change for Persons With Type 2 Diabetes, Part 2: One-Year Results From the Norwegian Randomized Controlled Trial RENEWING HEALTH. JMIR Mhealth Uhealth. 2014 Dec 11;2(4):e57. PMID: 25499872

63. Torbjørnsen A, Jenum AK, Småstuen MC, Arsand E, Holmen H, Wahl AK, et al. A low-intensity mobile health intervention with and without health counseling for persons with type 2 diabetes, part 1: baseline and short-term results from a randomized controlled trial in the Norwegian part of RENEWING HEALTH. JMIR Mhealth Uhealth 2014;2(4):e52. PMID: 25499592.

64. Holmen H, Wahl A, Torbjørnsen A, Jenum AK, Småstuen MC, Ribu L. Stages of change for physical activity and dietary habits in persons with type 2 diabetes included in a mobile health intervention: the Norwegian study in RENEWING HEALTH. BMJ Open Diabetes Res Care 2016;4(1):e000193. PMID: 27239317.

65. Agarwal P, Mukerji G, Desveaux L, Ivers NM, Bhattacharyya O, Hensel JM, et al. Mobile App for Improved Self-Management of Type 2 Diabetes: Multicenter Pragmatic Randomized Controlled Trial. JMIR mHealth uHealth. 2019 Jan;7(1):e10321. PMID: 30632972.

66. Adu MD, Malabu UH, Callander EJ, Malau-Aduli AE, Malau-Aduli BS. Considerations for the Development of Mobile Phone Apps to Support Diabetes Self-Management: Systematic Review. JMIR mHealth uHealth. 2018 Jun;6(6):e10115. PMID: 29929949.

67. Riley WT, Rivera DE, Atienza AA, Nilsen W, Allison SM, Mermelstein R. Health behavior models in the age of mobile interventions: are our theories up to the task? Transl Behav Med. 2011 Mar;1(1):53-71. PMID: 21796270.

68. Tchero H, Kangambega P, Briatte C, Brunet-Houdard S, Retali G-R, Rusch E. Clinical Effectiveness of Telemedicine in Diabetes Mellitus: A Meta-Analysis of 42 Randomized Controlled Trials. Telemed J e-health Off J Am Telemed Assoc. 2019 Jul;25(7):569-83. PMID: 30124394.

69. Waschkau A, Uebel T, Steinhäuser J. Diabetes treatment 2.0: telemedicine. Internist (Berl). 2019 Sep;60(9):917-24. PMID: 31346638

70. Stanton CA, Keith DR, Gaalema DE, Bunn JY, Doogan NJ, Redner R, et al. Trends in tobacco use among US adults with chronic health conditions: National Survey on Drug Use and Health 2005-2013. Prev Med (Baltim). 2016 Nov;92:160- PMID: 27090919

71. Huckvale K, Adomaviciute S, Prieto JT, Leow MK-S, Car J. Smartphone apps for calculating insulin dose: a systematic assessment. BMC Med. 2015 May;13:106. PMID: 25943590.

\section{Figures}



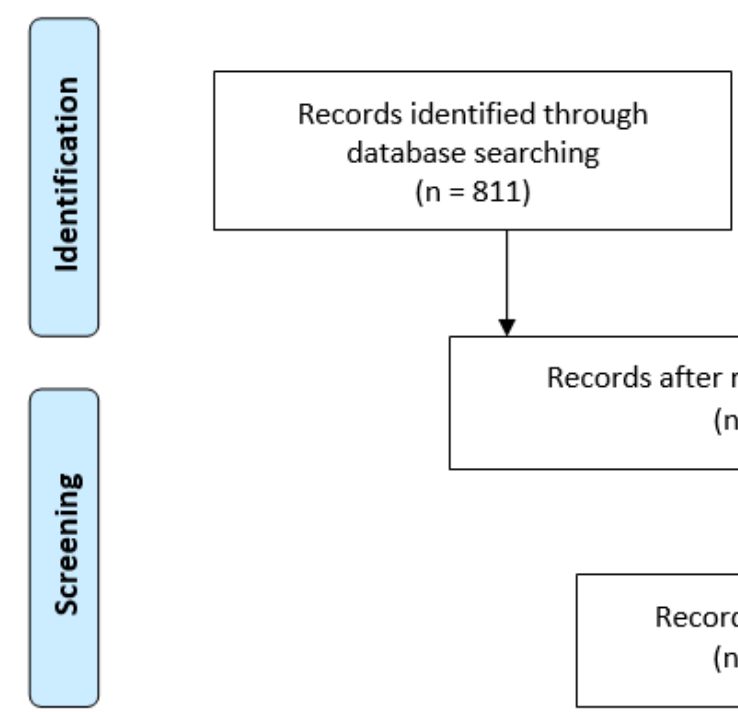

Records after removing duplicates

Additional records identified through specialized journals $(n=811)$

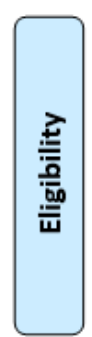

$$
\text { ( } n=798)
$$$$
(n=10)
$$

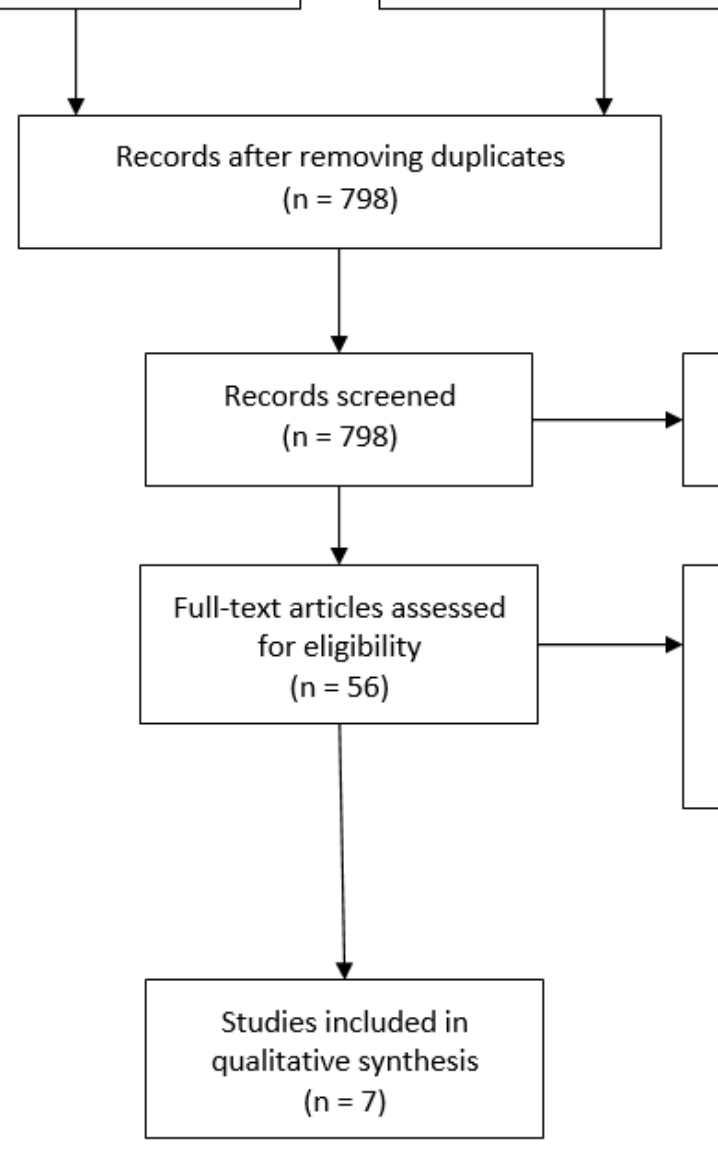

Records excluded by title and abstract $(n=742)$

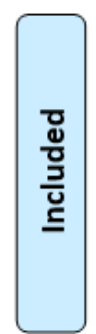

Full-text articles excluded for:

- $\quad(n=39)$ It did not comply with inclusion criteria.

- $\quad(n=10)$ Low metodological quality.

\section{Figure 1}

Flow diagram of PRISMA 

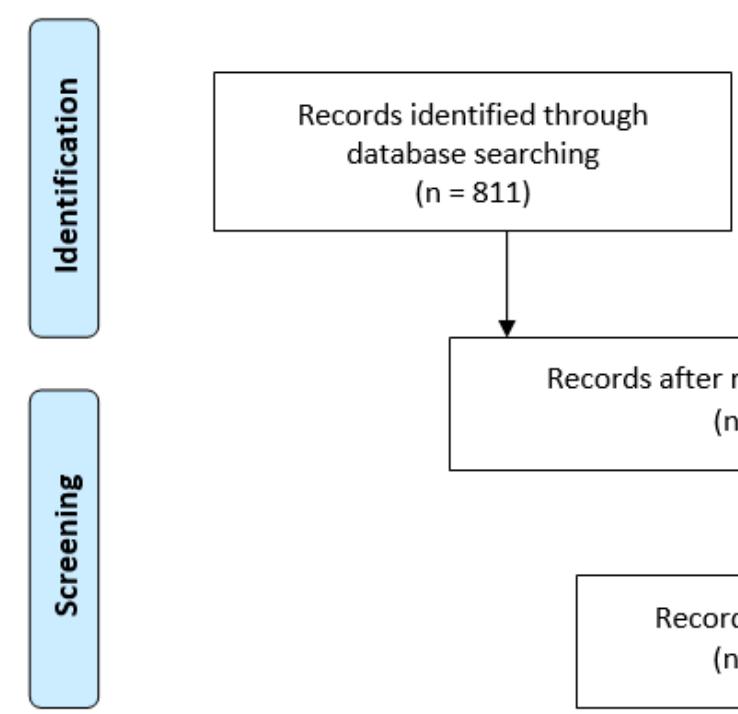

Records after removing duplicates

$(n=798)$
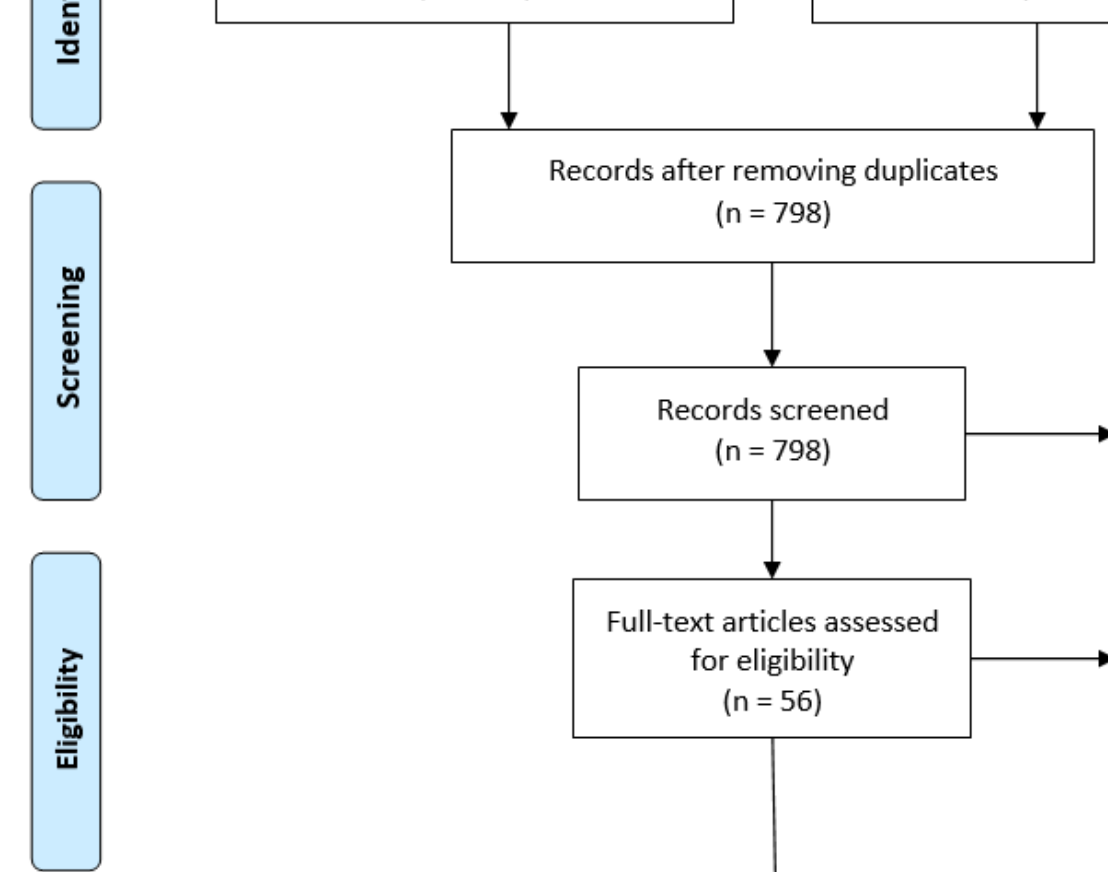
through specialized journals $(n=10)$

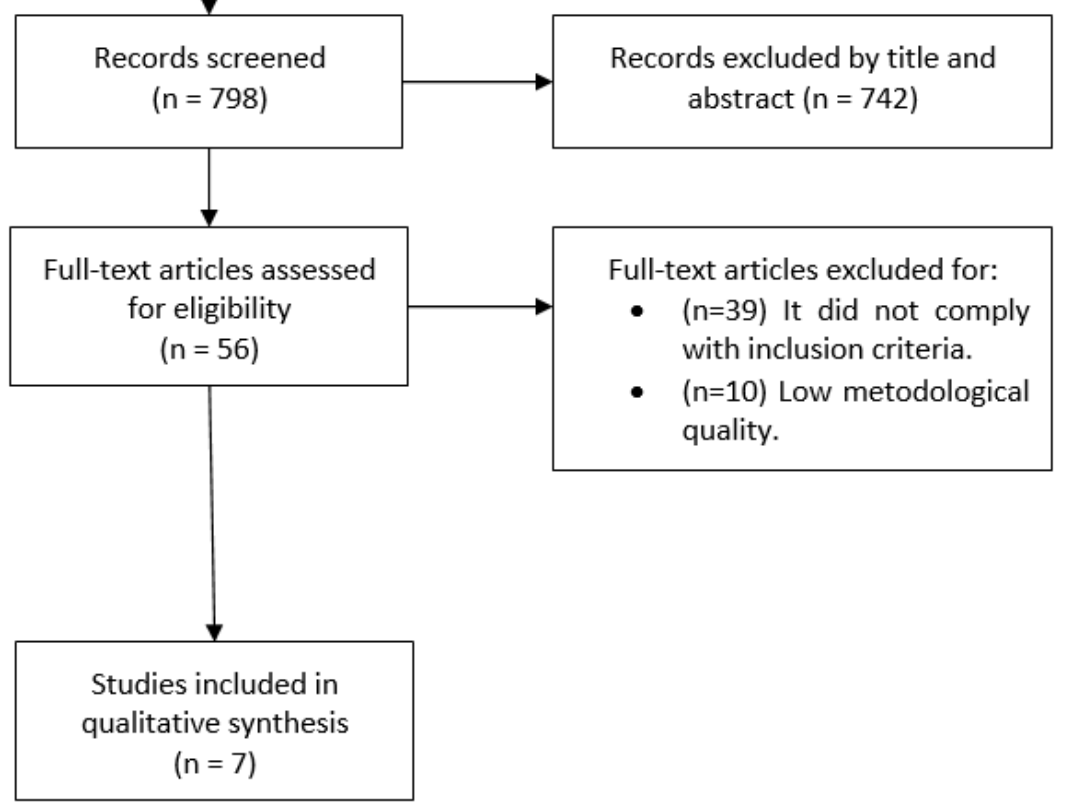

\section{Figure 1}

Flow diagram of PRISMA

\section{Supplementary Files}

This is a list of supplementary files associated with this preprint. Click to download.

- MultimediaAppendix1.SearchstrategyinMedline.doc

- MultimediaAppendix1.SearchstrategyinMedline.doc 